\title{
Primary Care Follow Up Post Mitral Valve Surgery at Ambulatory Clinic
}

Andrew Yin, $M D$

\section{Case Presentation}

A 67 year-old Cantonese speaking male was seen at primary care clinic with complaints of dyspnea on exertion and decreased exercise tolerance. His medical history includes rheumatic heart disease, tricuspid regurgitation, tricuspid repair three months ago, mitral regurgitation, mitral valve replacement with a bioprosthetic valve three months ago, atrial fibrillation on anticoagulation therapy, permanent pacemaker implantation, severe pulmonary hypertension, benign prostatic hypertrophy, gastroesophageal reflux disease, and iron deficiency anemia. The patient reports that in the last two weeks, he has been unable to ambulate more than two blocks limited by shortness of breath, which represents an acute change. He also complains of bilateral lower extremity edema. He reports that two weeks after his mitral valve replacement, his exercise tolerance was dramatically improved, and he was able to ambulate more than two blocks without difficulty.

He denies associated cough, lightheadedness, dizziness, chest discomfort, abdominal discomfort, nausea or vomiting. $\mathrm{He}$ reports occasional palpitations that are not associated with his episodes of dyspnea on exertion. Dyspnea resolves with rest in minutes. He denies recent fevers, sweats, and chills. Medications include metoprolol $25 \mathrm{mg}$ twice a day, warfarin $4 \mathrm{mg}$ daily, furosemide $40 \mathrm{mg}$ daily, enteric-coated aspirin $81 \mathrm{mg}$ daily, sustained-release potassium chloride $20 \mathrm{mEq}$ daily, ferrous sulfate $300 \mathrm{mg}$ three times per day, colace $100 \mathrm{mg}$ twice per day, sennosides $8.6 \mathrm{mg}$ daily and omeprazole $20 \mathrm{mg}$ daily

On physical examination, the patient was alert and oriented to person, place, and time and not in acute distress, afebrile, with a heart rate of 76 beats/minute, blood pressure of $90 / 60 \mathrm{~mm}$ $\mathrm{Hg}$, respiratory rate 14 breaths/minute. Pupils were equal and reactive to light, and extraocular muscles were intact. There were no carotid bruits bilaterally, and jugular venous pressure was estimated at $16 \mathrm{~cm}$ of water. His lungs were clear to auscultation bilaterally with no wheeze, no crackles, nor rales. Cardiac exam demonstrated a midline sternal surgical scar, an irregularly irregular heart rhythm, no murmurs, gallops, nor rubs. Abdominal exam demonstrated a soft, nontender, nondistended abdomen with normoactive bowel sounds and no organomegaly. His extremities did not demonstrate lower extremity edema, clubbing, or cyanosis, and his pulses were $2+$ radial, $2+$ femoral, and $2+$ dorsalis pedis bilaterally. Neurologic exam did not reveal any focal deficits.

Electrocardiogram demonstrated atrial fibrillation with a ventricular response rate of 90 beats per minute, normal axis, peaked $\mathrm{T}$ waves, and no acute changes suggestive of ischemia. Routine basic metabolic panel done five days prior to his office visit was remarkable for a potassium of $5.0 \mathrm{mEq} / \mathrm{L}$.
The patient also had an appointment with his cardiologist for evaluation of his symptoms. After the evaluation he was admitted directly to hospital for further evaluation of his new symptoms of congestive heart failure. His admission labs were unremarkable with the exception of B-type natriuretic peptide of $1463 \mathrm{pg} / \mathrm{mL}$. His admission chest $\mathrm{x}$-ray was suggestive of a left pleural effusion, cardiomegaly and pulmonary edema.

Transthoracic echocardiogram revealed the bioprosthetic valve in the mitral position had its ventricular side tilted toward the interventricular septum. There was no evidence of rocking of the mitral prosthesis. The peak mitral valve gradient was $13 \mathrm{~mm}$ of $\mathrm{Hg}$. The mean transmitral valve gradient was $4 \mathrm{~mm}$ of $\mathrm{Hg}$. The estimated mitral valve area was $2.6 \mathrm{~cm}^{2}$ which is described as normal in the setting of a prosthetic valve. There was a moderate paravalvular regurgitant leak between the prosthesis and lateral wall of the left ventricle (LV). The effective regurgitant orifice area of the paravalvular leak is estimated at $0.33 \mathrm{~cm}^{2}$. There was evidence of mild aortic regurgitation, mild left atrial enlargement and an overall severely decreased LV systolic function with segmental wall motion abnormalities. The distal half of the LV is akinetic. The proximal walls were severely hypokinetic. LV ejection fraction was estimated to be $20 \%$, and there was right ventricular (RV) enlargement with decreased function. There was no evidence of vegetations on the valves or pacemaker leads. Compared with an echocardiogram done one month following replacement, of his mitral valve, there was interval deterioration of LV function. The paravalvular leak appeared unchanged.

Computed tomography of the thorax without contrast demonstrated an irregular consolidation in right upper lobe which was indeterminate, but most likely represented atelectasis and/or pneumonitis, bilateral pleural effusions, a left lower lobe consolidation and an enlarged left heart post mitral and tricuspid valvular repair.

Cardiothoracic surgery was consulted. Due to the malpositioning of the prosthetic valve and evidence of worsening ventricular function, it was decided that the patient would need another operation to replace his prosthetic mitral valve.

\section{Discussion}

Heart valve surgery is still considered one of the most important advances in patients with symptomatic, severe valvular heart disease. ${ }^{1}$ Annually, there are an estimated 42,000 mitral valve procedures in the United States. ${ }^{2}$ It is important to appreciate that replacement of a diseased valve is not a cure for the valvular disease process, but rather an exchange of the native disease for a new set of complications that are associated to the new valve prosthesis. ${ }^{3,4}$ For this reason, the frequency and intensity of follow up and evaluation of prosthetic valves is similar to the follow up of newly diagnosed native valve disorders. The American College of Cardiology (ACC) and the American 


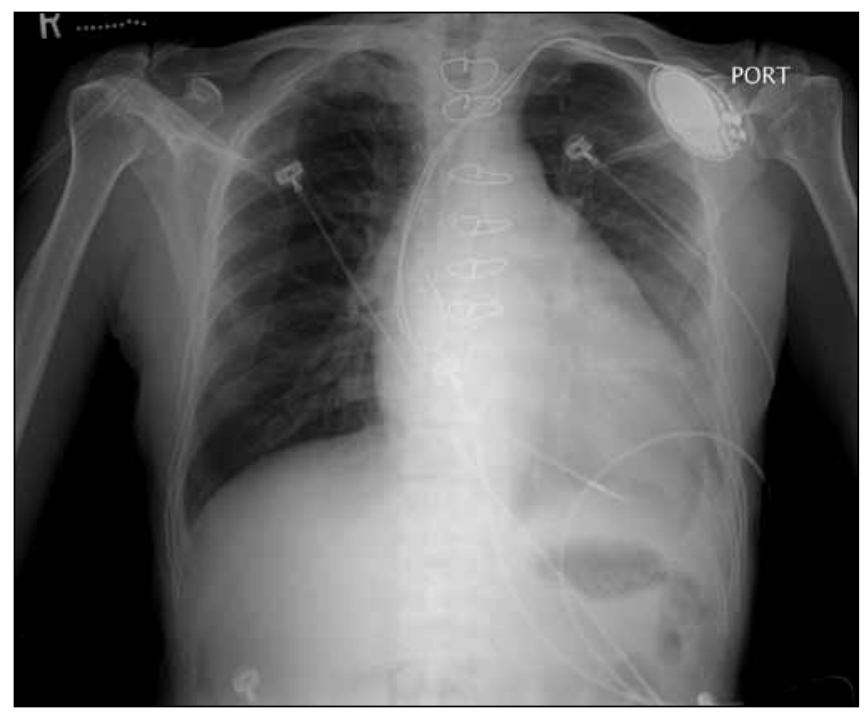

Figure 1. The patient's chest radiograph shows cardiomegaly, pulmonary vascular congestion, developing consolidation in left lower left lower lobe, a left-sided dual chamber pacemaker, midline sternotomy wires, and evidence of a tricuspid annuloplasty ring.

Heart Association (AHA) Task Force recommends the following guidelines for evaluation and management of patients after valve replacement: ${ }^{3}$

The first postoperative outpatient evaluation should be two to four weeks after hospital discharge. By this time, the patient's physical capabilities and expected improvement in functional capacity can be assessed. The focus of this initial evaluation is toassessfor infection and myocardialinfarction, an assessment of function of the prosthetic valve, electrical conduction and other valvular disorders. Follow up should include an interval post-op history, physical examination, an electrocardiogram, complete blood count, electrolytes, an INR as indicated for management of anticoagulation therapy and a transthoracic Doppler echocardiogram. Echocardiography can provide information about prosthesis stenosis or regurgitation, valve area, pulmonary hypertension, LV or RV hypertrophy, LV and RV size and function, pericardial effusion and thickening, perivalvular regurgitation and assess function of the other heart valves. This initial echocardiogram is important to establish baseline characteristics of the prosthetic valve if one was not yet done prior to discharge.

Follow up of asymptomatic patients should be conducted annually. No further echocardiographic testing is required after the initial postoperative evaluation in patients who are stable and do not have symptoms or clinical evidence of LV dysfunction, prosthetic valve dysfunction, or dysfunction of other heart valves. Reevaluations should be conducted if there is any change in clinical status or development of new symptoms. An echocardiogram is indicated in any patient whenever there is evidence of a new murmur or change in clinical status, when there are questions about prosthetic valve integrity and function, and when there are concerns about ventricular function. If the patient develops valvular regurgitation, close follow up with echocardiography every three to six months is indicated.

Patients who do not improve after surgery or who later show deterioration of functional capacity should be evaluated with an echocardiogram and, if necessary, transesophageal echocardiography and cardiac catheterization with angiography to determine the cause.

Prosthetic heart valves are associated with a number of complications including structural failure or deterioration, valve obstruction from thrombosis, systemic embolization, bleeding, endocarditis, impaired LV systolic function, hemolytic anemia, and other infections. Perivalvular leakage, a form of structural failure, is common. Severe perivalvular regurgitation maybe inaudible on physical exam. Most are detected intraoperatively during the original valve surgery. Rates are reported to be between 18 to $48 \%{ }^{5,6}$ The majority of perivalvular leaks are trivial and are not clinically significant and do not progress. ${ }^{5,6}$ Our patient's work up revealed that not only was his prosthetic mitral valve malpositioned, but also his echocardiogram showed an interval decline in his left ventricular systolic function three months after operation.

Re-operation to replace a prosthetic heart valve is a considered serious clinical event. The ACC/AHA Task Force recommends re-operation for moderate to severe prosthetic dysfunction (structural and nonstructural), dehiscence, and prosthetic endocarditis. Other indications include patients who have recurrent thromboembolism, severe intravascular hemolysis, severe recurrent bleeding from anticoagulant therapy, and thrombosed prosthetic valves. Stable patients without prosthetic valve endocarditis under many circumstances who requires reoperation are only slightly greater operative risk compared to the risk of the initial surgery. In patients with catastrophic prosthetic valvular dysfunction, surgery is clearly indicated and urgent. Patients without endocarditis or severe prosthetic valve dysfunction require careful hemodynamic evaluation, and the decision about reoperation should then be based on hemodynamic abnormalities, symptoms, ventricular function, and the natural history of the particular prosthesis. ${ }^{3}$

In summary, patients who undergo mitral valve replacement for treatment of their disease are not completely cured of valvular heart disease and must be followed with the same care as patients with native valve disease. ${ }^{7}$ A number of post-operative complications are common and may develop early or late in the post-operative period. The ACC/AHA Task Force on the management of valvular heart disease recommends close annual 
evaluation and a baseline echocardiogram initially to monitor valve structure and function. Though patients generally follow up with subspecialists for evaluation of their prosthetic valves, our patient sought all of this medical and post-operative care with his primary care physician because of because of language and cultural barriers. It is therefore critical that primary care physicians also recognize the signs and symptoms of the serious complications related to prosthetic valves.

\section{Hospital Course}

On day five of his hospital course, the patient was brought to the operating room for a replacement of his prosthetic valve. It was discovered that the patient had three major defects. The first was a $0.5 \mathrm{~cm}$ defect near the mitral-aortic continuity with the surgical pledgets having torn through the annulus with subsequent partial dehiscence of the prosthetic mitral valve. The tissue around the dehiscence was friable and could not be repaired. The prosthesis was excised and replaced. The second defect was a linear tear below the left coronary cusp of the aortic valve that disrupted the aortic ventricular continuity. The defect could not be repaired and thus was also replaced. Lastly, the third defect was a tear of the pericardium which was repaired using a sheet of bovine pericardium. Intraoperatively, the patient continued to have significant bleeding from behind the aortic root and was treated with various hemostatic agents including Biogel and Surgicel. He required multiple vasoactive medications and required multiple units of blood products but continued to be hemodynamically unstable with a pressure of 55/30 and a heart rate of 60 beats per minute. His cardiac rhythm on telemetry demonstrated multiple episodes of ventricular tachycardia. His condition and gravely poor prognosis was discussed with his family, and it was decided that the medical team would halt all attempts at cardiac resuscitation. The patient expired shortly after with his family at his bedside.

His family consented to an autopsy which revealed evidence of rheumatic heart disease, RV and LV dilatation and hypertrophy, and left atrial dilatation. His lungs had signs of acute and chronic passive congestion. Interestingly, there were histological signs of adenocarcinoma in his prostate. His final cause of death was determined to be due to pericardial and mediastinal hemorrhage status post open heart surgery for mitral and aortic valve replacement.

\section{References}

1. Rahimtoola SH. Lessons learned about the determinants of the results of valve surgery. Circulation 198878 1503-7.

2. Gunderson TJ, Nelson TB, Sullivan AJ. Heart valve market overview. Minneapolis, Minn: US Bancorp Piper Jaffray; 2003.

3. Bonow, RO, Carabello, BA, Chatterjee, $\mathrm{K}$, et al. ACC/AHA 2006 guidelines for the management of patients with valvular heart disease. A report of the American College of Cardiology/American Heart Association Task Force on Practice Guidelines (Writing committee to revise the 1998 guidelines for the management of patients with valvular heart disease). J Am Coll Cardiol 2006; 48: e84-231.

4. Omran AS, Woo A, David TE, Feindel CM, Rakowski H, Siu SC. Intraoperative transesophageal echocardiography accurately predicts mitral valve anatomy and suitability for repair. J Am Soc Echocardiogr. 2002; 15:950-7.

5. Rallidis, LS, Moyssakis, IE, Ikonomidis, I, Nihoyannopoulos, P. Natural history of early aortic paraprosthetic regurgitation: a five- year follow-up. Am Heart J 1999; 138:351.

6. O'Rourke, DJ, Palac, RT, Malenka, DJ, et al. Outcome of mild periprosthetic regurgitation detected by intraoperative transesophageal echocardiography. J Am Coll Cardiol 2001; 38:163.

7. Rahimtoola SH. Early valve replacement for preservation of ventricular function? Am J Cardiol 1977;40:472-5.

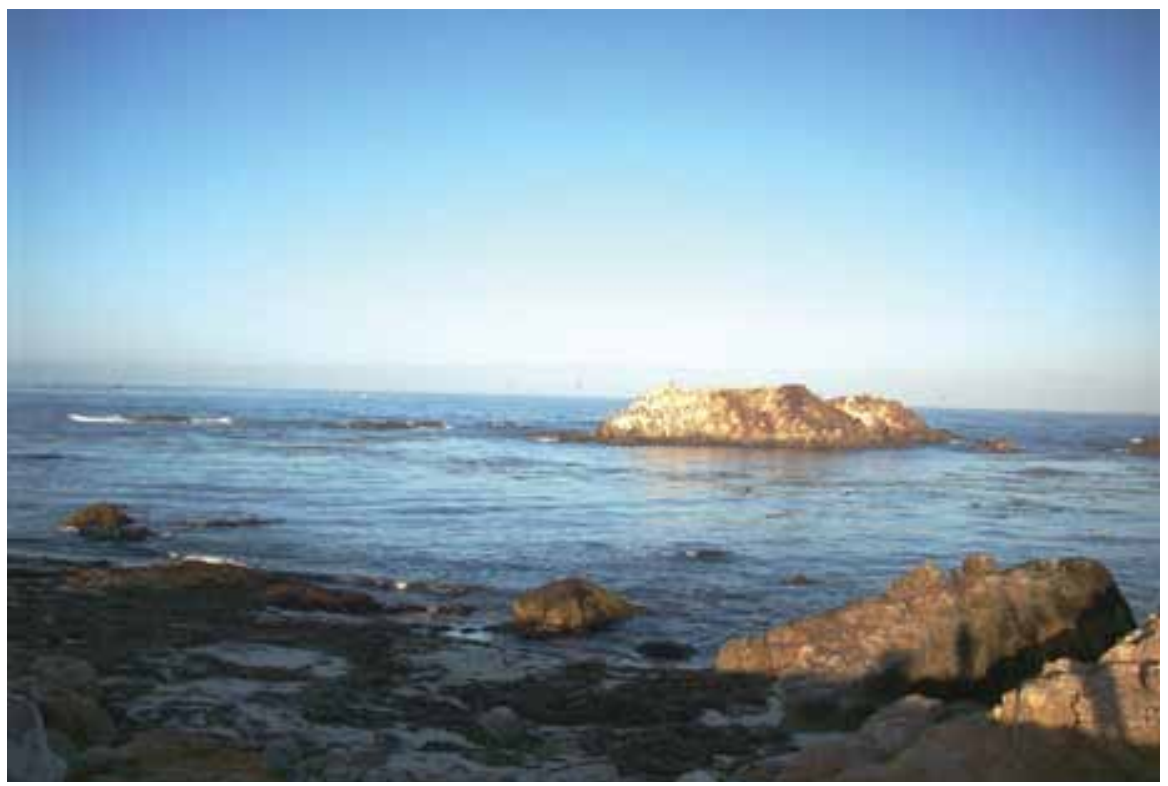

